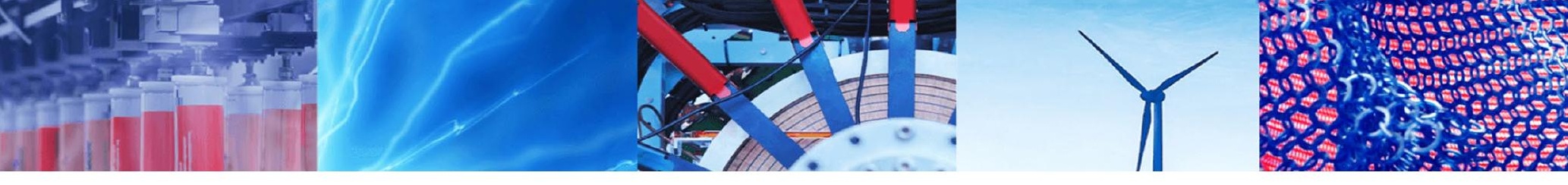

Research Article

\title{
Polymeric colloidal nanocarriers entrapped with Centella asiatica extract
}

\author{
Amanda Gomes Marcelino Perez ${ }^{1}$. Jocasta Mileski Machado ${ }^{1} \cdot$ Kelly Cristina Manhani ${ }^{2} \cdot$ Patrícia Leo $^{2}$. \\ Peky Noriega ${ }^{3} \cdot$ Maria Helena Ambrosio Zanin ${ }^{2}$ (i)
}

Received: 3 August 2020 / Accepted: 13 October 2020 / Published online: 29 October 2020

(c) Springer Nature Switzerland AG 2020

\begin{abstract}
Nanoparticle technology plays an important role in loading active ingredients and being able to increase their stability and performance. In this work, Centella asiatica extract, a plant with known dermatological and cosmetic applications, was incorporated into colloidal polymer nanocarriers, dispersed in silicophilic medium. Nanocarriers were prepared and characterized for particle size (Dynamic Light Scattering), morphology (Scanning Electron Microscopy) and entrapment efficiency of madecassoside. Physical and chemical stability of the encapsulated extract was monitored for 60 days of storage under specific conditions $\left(25\right.$ and $40^{\circ} \mathrm{C}$ ), quantifying the content of madecassoside by HPLC and the particle size distribution. In vitro safety assays, including halo diffusion, cell viability and cell transformation assay were evaluated for free and encapsulated extracts. Nanocarriers showed an average diameter around $210 \mathrm{~nm}$ (Polidispersity Index-PDI 0.2 ) and the entrapment efficiency of $97.7 \%$. The concentration of madecassoside remained stable over time, indicating that the nanocarriers proposed here were able to protect madecassoside against degradation. In addition, nanocarriers showed non-cytotoxic effects and did not induce cell transformation.
\end{abstract}

Keywords Centella asiatica extract $\cdot$ Nanoparticles $\cdot$ Polymeric nanocarriers $\cdot$ Madecassoside

\section{Introduction}

Centella asiatica (L.) Urban (C. asiatica), also known as Gotu Kola, is a small perennial plant [1] used in traditional medicine in different parts of the world, sucha as Southeast Asia, India, China and Indonesia. The main constituents responsible for pharmacological activities are the pentacyclic triterpenes (asiatic acid, madecassic acid, asiaticoside, and madecassoside) [2]. C. asiatica has significantly drawn the attention of scientific groups in the recent years due to its broad range of applications, such as in skin diseases [3], wound healing [4], anti-aging and skin moisturizing [5], hepatoprotective [6], anticancer, antibacterial, antifungal, anti-inflammatory [3], neuroprotective [7, 8], antioxidant
$[9,10]$, antidiabetic [11], cognitive function enhancement [12]. The literature has numerous success studies indicating the cosmetic potential of Casiatica in the treatment of wrinkles and photoaging skin $[13,14]$, cellulite and striae $[15]$, and as a skin moisturizing $[4,16]$. The skin aging is a multi-factorial phenomenon induced by intrinsic (chronological aging) and extrinsic factors (like ultraviolet damage, pollution, harsh weather, and cigarette smoke) [17], which the chronological aging leads to a decrease in the number of fibroblasts and, consequently, the synthesis of collagen and elastin also decreases, resulting in skin wrinkles and loss of elasticity $[18,19]$. Further, the total hyaluronic acid (HA) level in the epidermis diminishes markedly [20] and the skin becomes drier due to the loss of sebaceous

$\triangle$ Maria Helena Ambrosio Zanin, mhzanin@ipt.br|' Support Foundation of Institute for Technological Research (FIPT), Bionanomanufacturing Nucleus, Eng. Heitor Antônio Eiras García Avenue, 448, Jardim Esmeralda, São Paulo, SP 05588-000, Brazil. ${ }^{2}$ Institute for Technological Research (IPT), Bionanomanufacturing Nucleus, Prof. Almeida Prado Avenue, 532, Butantã, São Paulo, SP 05508-901, Brazil. ${ }^{3}$ Nanofitotec, Lauro Vanicci Street, 1020, Parque Rural Fazenda Santa Candida, Campinas, SP 13087-548, Brazil. 
glands in the dermis. Sun exposure is the principal cause of extrinsic aging, which UV radiation is absorbed, generating reactive oxygen species (ROS) inducing the synthesis of proteolytic enzymes such as matrix metalloproteinases (MMPs), responsible for the breakdown of the extracellular matrix, resulting in wrinkles [21,22].

In vitro studies have demonstrated the anti-hyaluronidase and anti-elastase potential of the $C$ asiatica extract, besides the matrix metalloproteinase-1inhibition (MMP-1) [5]. The $C$. asiatica extract can stimulate collagen synthesis, upregulate aquaporins -3 (AQCP3) expression [14], and promote free radical scavenging activity $[10,23]$. Besides that, he extract has been shown to be an efficient skin protector against UV radiation [13], comparable with OMC (octyl methoxy cinnamate) in its protection against UVB absorbance, showing its potential as a natural protector against UVB damage [10]. Clinical studies have shown antinflammatory, skin hydration and anti-wrinkle potential activities of $C$. asiatica extract $[13,24]$, showing its great potential of application as a cosmeceutical product.

Nanotechnology combined with cosmeceuticals nowadays is a strategy that can bring many benefits, such as controlled release of the active substances, increased permeation through the skin, greater physical stability, among others [25]. This strategy may be especially important in the case of water-soluble plants extracts, due to its difficulty of permeation through the skin, which naturally has hydrophobic characteristics $[26,27]$.

Some studies have been carried out to protect C. asiatica extract, improving its stability and bioavailability [26, 28]. Yongsirasawad et al. [29] prepared C. asiatica extractloaded Gelatin Nanoparticles (CGNP) by using gelatin one-step desolvation method, achieving an entrapment efficiency around $36 \%$ and an antibacterial activity three times higher of the CGNP compared to the crude extract. Moreover, bovine serum albumin (BSA) nanoparticles containing CA extracts, prepared by an adapted desolvation method reached encapsulation efficiency of around $90 \%$ and showed the potential of the nanoparticles to protect CA extract against hydrolysis and biotransformation [30].

In addition, CA extract also is also being vehicled in polimeric films for topical application [31]. Electrospun gelatin fiber containing methanolic CA extract cross-linked with glutaraldehyde vapor, resulted in fibers with average diameters between 226 and $232 \mathrm{~nm}$, stable in an aqueous medium and able to promote a controlled release of asiaticoside [32]. In another study, Monton et al. [33] prepared film forming polymeric dispersion (FFPD) containing C. asiatica extract using Eudragit ${ }^{\circ}$ RS $30 \mathrm{D}$ and Eudragit ${ }^{\circ}$ RL 30D as the film forming agents, resulting in a loading efficiency about $100 \%$ and achieving formulations able to promote sustained release and accumulation of madecassoside and asiaticoside in the skin layers, with no detection of these phytoactives in the release medium, indicating the safety of the formulation in not permeating through the dermis. However, most of these studies use organic solvents in their production processes or in the chemical cross-linking agents and several them still use raw materials derived from animals such as gelatin in the preparation of nanostructures, which has been avoided by the cosmetic sector.

In this context, in this work, C Asiatica extract was entrapped in polymeric colloidal nanocarriers (PNC-CA) aiming to improve its stability and control the madecassoside release. The nanocarriers were prepared by using a hydrophilic polymer dissolved in an aqueous phase and emulsified in a silicone phase containing the surfactant, through a process that avoids the use of organic solvents or toxic crosslinking agents. Characterization of the PNCCA included particles size measurement by photon correlation spectroscopy (PCS); morphology by Scanning Electron Microscopy (SEM); entrapment efficiency of madecassoside; stability of PNC-CA over time and in vitro safety tests.

\section{Materials and methods}

\subsection{Materials}

Centella asiatica glycolic extract was purchased from Mapric, São Paulo, Brazil; polydimethylsiloxane fluid DC 200, 100 CP (Focus Química); starch purity gum 1773 (Ingredion, Canada); - Cyclopentasiloxane SF 1540 silicone (Momentive), sodium chloride (Dinâmica), Madecassoside and Asiaticoside (Sigma Aldrich), DMEM-7777 (Sigma Aldrich). The NCTC-clone L929 mouse connective tissue strain from the American Type Culture Collection [ATCC(CCL1) was purchased from the Adolfo Lutz Institute cell bank (Cultcel) and the Balb / c 3 T3 strain was purchased from the Rio de Janeiro cell bank (BCRJ-UFRJ).

\subsection{Preparation of polymeric colloidal nanocarrier entrapped with Centella asiatica extract}

Polymeric colloidal nanocarriers entrapped with C. asiatica extract (PNC-CA) was prepared according to the procedure described by Cerize et al. [34]. Briefly, the process was carried out basically in three steps. Step 1: pre-emulsion formation by dispersing the internal phase (C. asiatica extract, starch, sodium chloride and water) in the external phase, Polydimethylsiloxane fluid and SF 1540 silicone; Step 2: nanoemulsification of the pre-emulsion formed in Step 1, performed in a high pressure homogenizer at 1000 bar in two cycles; Step 3: extraction of the inner aqueous phase, under controlled pressure $(180-210 \mathrm{mmHg})$ and temperature of $50^{\circ} \mathrm{C}$ in order to obtain the PNC-CA. 


\subsection{Particle size distribution and polydispersity index (PDI)}

The particle size distribution and polydispersity index (PDI) were determined based on the analytical technique of Dynamic Light Scattering using the equipment Nanoplus (Particulate Systems). The samples $(50 \mu \mathrm{L})$ were dispersed in $6 \mathrm{~g}$ of fluid silicon SF 1202 containing 2\% of emulsifier silform 60-A and homogenized and transferred to the sample cell. The measurements were done in triplicate and the software ZetaSizer Version 6.20 was used to manage the test and treat the data.

\subsection{Morphology by scanning electron microscopy}

Morphology of PNC-CA was evaluated by Scanning Electron Microscopy (Field Electrons Gum-Scanning Electron microscope-FEG-SEM, model Quanta 3D, FEI). The samples were prepared prior to the analysis: PNC-CA was filtered through a cellulose ester membrane (porosity $0.2 \mu \mathrm{m}$ ) in a vacuum system. The membrane was then washed with cyclohexene to remove the silofilic medium and preserve the nanocarriers. These membranes were then placed in desiccator under vacuum for drying for 24 h. After gold coating (Desc IV, Denton), samples were visualized with the scanning electron microscope (SEM) with an accelerating voltage ranging between 10 and $20 \mathrm{kV}$.

\subsection{Biomarkers content: high performance liquid chromatography (HPLC) analysis}

Madecassoside and asiaticoside have been chosen as markers for $C$. asiatica extract. Identification and quantification of madecassoside in the PNC-CA and C. asiatica extract were determined by high performance liquid chromatography (HPLC). The methodology used was developed by Institute for Technological Research (IPT), based on the work of Rafamantanana et al. [35]. During the development of the HPLC method, different proportions of mobile phase were tested, as well as the addition of trifluoroacetic acid and methyl tert butyl ether to the aqueous and organic phase, according to previous work of Schaneberg et al [36]. Using the Shim-Pack GIST column, the most efficient method was adapted to work of Rafamantanana et al. [35], and the parameters of Linearity, Limit of Detection (LOD) and Limit of Quantification (LOQ) were considered in order to ensure the quality and reliability of the results.

The quantification of madecassoside was performed by High Performance Liquid Chromatograph (HPLC), model SPD-M20A, UV Vis detector (Shimadzu). The column used was Shim-Pack GIST-Shimadzu $(5 \mu \mathrm{m}-\mathrm{C} 18$,
$4.6 \mathrm{~mm} \times 250 \mathrm{~mm}$ ) and the detection wavelength was set at $206 \mathrm{~nm}$. The mobile phase was a gradient of acetonitrile:water (0.01-15 min., 20:80; 15-25 min., 35:65; 25-30 min., 65:35; 25-40 min., 20:80) and the flow rate was $1.0 \mathrm{~mL} / \mathrm{min}$. Prior to injection, samples and standards were filtered through a $0.22 \mu \mathrm{m}$ membrane syringe filter (PTFE-hidrophylic). The injection volume was $20 \mu \mathrm{L}$ and it was performed three injections for each standards and samples. The concentrations of madecassoside in the samples were estimated from the standard curve. Asiaticoside was not identified in the extract. The standard curve was prepared from a stock solution of madecassoside $\left(1000 \mathrm{mg} \mathrm{L}^{-1}\right)$, prepared with standard madecassoside (from Sigma Aldrich $\geq 95 \%$ ) using ultrapure water. From this stock solution the calibration curve was prepared, at concentrations of $8 ; 10 ; 20 ; 25 ; 30 ; 40$ and $50 \mathrm{mg} \mathrm{L}^{-1}$. The linear equation and LOD and LOQ of madecassoside were also determined.

\subsubsection{Preparation of PNC-CA samples for madecassoside quantification}

In order to eliminate the effect of the matrix in which the nanocarriers were dispersed (silicone), $8 \mathrm{~g}$ of the formulation was centrifuged at $15.115 \times g$ for $30 \min \left(25^{\circ} \mathrm{C}\right)$. Subsequently, part of the supernatant was removed, and the recovered decanted material was used in the determination of madecassoside content and encapsulation efficiency. Thus, to perform the calculations, a correction factor (FC) was applied:

$F C=\frac{\text { Mass of decanted material }(\mathrm{g})}{\text { Total sample mass }(\mathrm{g})}$

where: mass of decanted material is the total mass of product-the mass of supernatant removed after the centrifugation process.

\subsubsection{Total madecassoside content (Madeca $\left.{ }_{\text {total }}\right)$}

About $0.25 \mathrm{~g}$ of the material decanted, obtained as described in Sect. 2.5.1. was added in a $2 \mathrm{~mL}$ micro tubes with $1 \mathrm{~mL}$ of methanol. The sample was vortexed for $1 \mathrm{~min}$, so that the methanol could break the nanocarriers, releasing the active substance in the medium. This mixture was centrifuged at $2.236 \times g$ for $5 \mathrm{~min}$ and supernatant was transferred to another tube. The precipitate was washed twice more with $1 \mathrm{~mL}$ of methanol and centrifuged at $8.944 \times g$ and $15.115 \times g$ for 5 min each.

The collected supernatants were concentrated in a vacuum system $\left(40{ }^{\circ} \mathrm{C}\right)$ and the concentrated material was resuspended in $1 \mathrm{~mL}$ of methanol, filtered through a $0.22 \mu \mathrm{m}$ hydrophilic PTFE filter and analyzed by HPLC for 
madecassoside quantification, according to the method described in Sect. 2.5 .

Total concentration of madecassoside in the final product ([Madeca $\mathrm{Total}_{\text {] }}$ in $\mathrm{mg} \mathrm{kg}^{-1}$ ) was calculated using Eq. (2):

$\left[\right.$ Madeca $\left._{\text {Total }}\right]=\frac{\left(\left[C E_{\text {madeca }}\right] \times F V\right)}{W_{\text {sample }}} \times F C$

where: $\left[C E_{\text {madeca }}\right]$ is the madecassoside concentration obtained by HPLC (in $\mathrm{mg} \mathrm{L}^{-1}$ ); FV is the final volume of the resuspended concentrated material and $W_{\text {sample }}$ is the sample weight $(\mathrm{mg})$ in the eppendorf tube.

To obtain the final result of the madecassoside concentration in Eq. (2), it was necessary to multiply by the correction factor (Eq. 1) due to the sample concentration, as mentioned in item Sect. 2.5.1.

\subsubsection{Entrapment efficiency}

In order to quantify only the madecassoside entrapped into the nanocarrier structure, the precipitate obtained in Sect. 2.5.1 was washed with ethyl acetate and centrifuged again at $8.944 \times g$ for $15 \mathrm{~min}$, followed by removal of the supernatant. The precipitate collected were washed more twice with ethyl acetate and dried at $40{ }^{\circ} \mathrm{C}$ for $3 \mathrm{~h}$ in an oven. Dried material was suspended in water, vortexed and placed in ultrasound bath until total solubilization. The obtained mixture was filtered through a $0.22 \mu \mathrm{m}$ hydrophilic PTFE filter and analyzed by HPLC according to methodology described in Sect. 2.5, thus obtaining only the concentration of madecassoside into the nanostructure.

The entrapment efficiency (EE, \%) was calculated according to Eq. (3), dividing the concentration of madecassoside into the nanocarriers $\left[\right.$ Madeca $\left._{\text {encapsulated }}\right]$, in $\mathrm{mg} \mathrm{kg}^{-1}$ (after the correction applied in Eq. 1) by the total concentration of madecassoside [Madeca $\left.{ }_{\text {Total }}\right]$, in $\mathrm{mg} \mathrm{kg}^{-1}$.

$E E(\%)=\left(\frac{\left[\text { Madeca }_{\text {encapsulated }}\right]}{\left[\text { Madeca }_{\text {Total }}\right]}\right) \times 100$

\subsection{Evaluation of stability of PNC-CA: total madecassoside content and particle size distribution}

The stability study of PNC-CA considering the degradation rate of madecassoside in PNC-CA, besides the possible formation of impurities and the particle agglomerations were also evaluated. PNC-CA was stored at 25 and $40^{\circ} \mathrm{C}$ and the madecassoside content and particle size were evaluated at days 0, 7, 15, 30 and 60, as described in Sects. 2.5.2 and 2.3 , respectively.

\subsection{In vitro safety testing}

\subsubsection{Indirect-contact test: Agar diffusion}

This assay was performed according to the methodology described by Rogero et al. [37]. NCTC-929 cells $\left(1 \times 10^{5}\right.$ cells $\left.\mathrm{mL}^{-1}\right)$ were distributed in 6 wells plates and allowed to grow for $48 \mathrm{~h}$ in incubator at $37^{\circ} \mathrm{C}$, with $5 \%$ of $\mathrm{CO}_{2}$. After cell confluence, a preparation containing $1.8 \%$ of agar in DMEM medium with serum (2 times concentrated and containing $0.01 \%$ neutral red) was distributed over the cell monolayer. After solidification, non-toxic filter paper discs were soaked in C. asiatica extract solution and PNC-CA and deposited on the cells protected by the agar layer. As negative control it was used filter paper soaked in saline solution and as positive control, latex glove fragment. After $24 \mathrm{~h}$ of contact with the cells, the plates were observed macroscopically to verify the presence of a growth inhibition halo around the evaluated material and microscopically to observe the cellular aspects. The images were photo documented with the naked eye and through the microscope (Axiovert 135, Zeiss).

\subsubsection{Cell viability}

NCTC-929 cells $\left(1 \times 10^{5}\right.$ cells $\left.\mathrm{mL}^{-1}\right)$ were distributed in 96 wells plates $(100 \mu \mathrm{l} /$ well). After $24 \mathrm{~h}$ of incubation at $37^{\circ} \mathrm{C}$ with $5 \%$ of $\mathrm{CO}_{2}$, the culture medium was replaced by the test materials in different concentrations, diluted in DMEM medium with $5 \%$ of Serum Fetal Bovine (SFB). For the empty nanocarriers and PNC-CA the concentration was of $36 \%, 18 \%, 9 \%, 4.5 \%, 2.3 \%, 1.1 \%, 0.6 \%$ and $0.3 \%$ and for the $C$. asiatica extract the concentration was of $4.32 \%, 2.16 \%, 1.08 \%, 0.54 \%, 0.27 \%, 0.135 \%, 0.0675 \%$, $0.03375 \%$. The concentration of $C$. asiatica extract in the PNC-CA samples is corresponding to the concentrations of the free $C$. asiatica extract in this assay. All cells were incubated for further $24 \mathrm{~h}$ under the same conditions as described above. Therefore, cells were washed with phosphate buffer saline (PBS) and stained with neutral red vital dye (NR). This procedure was performed according to the Annex A (Neutral red uptake-NRU-cytotoxicity test) of ISO 10993-5: 2009-10 [38].

The tested material concentrations used here were based on the concentration of the PNC-CA in cosmetic bases, which we considered around $5 \%$. 


\subsubsection{Cell transformation assay (CTA)}

This assay was conducted according to the document Balb/c 3 T3 cell transformation assay (2010) [39]. Balb/c 3 T3 cells $2 \times 10^{3}$ cells $\mathrm{mL}^{-1}$ ) were cultivated in Petri dishes (diameter $=90 \mathrm{~mm}$ ) containing $10 \mathrm{~mL}$ of DMEM with $10 \%$ of SFB (approximately 180 cells $/ \mathrm{cm}^{2}$ ) and maintained in incubator at $37{ }^{\circ} \mathrm{C}$, with $5 \%$ of $\mathrm{CO}_{2}$ for $24 \mathrm{~h}$. After that, the medium was removed and test substances were added. Methylcolantrene reagent $\left(0.1 \mathrm{mg} \mathrm{mL}^{-1}\right)$ was used as positive control of cell transformation. For each material analyzed, $10 \mathrm{~mL}$ of culture medium, $1 \mathrm{~mL}$ of PNC-CA and $120 \mu \mathrm{L}$ of C. asiatica extract were used. After $72 \mathrm{~h}$, DMEM with tests substances were removed, replaced by fresh DMEM with $10 \%$ of SFB and incubated at $37^{\circ} \mathrm{C}$, with $5 \%$ of $\mathrm{CO}_{2}$ for 3 days.

After this time, the medium was removed again, replaced by DMEM with $2 \%$ of SFB and incubated at $37^{\circ} \mathrm{C}$, with $5 \%$ of $\mathrm{CO}_{2}$. This procedure was repeated twice week during 31-32 days. In the sequence, DMEM was removed and cells were fixed and stained with Giemsa 10\%. After Giemsa staining, the foci obtained were photographed and interpreted according to the catalog published by Sasaki et al. [40].
The concentrations of madecassoside in the analysis of content, entrapment efficiency and stability study were obtained through triplicate analysis of the samples under study. The results were based on the simple mean and standard deviation of the triplicate mean.

\section{Results}

\subsection{Particle size and polydispersity index (PDI)}

The mean particle size of the PNC-CA was $210.5 \mathrm{~nm}$ $( \pm 15.7 \mathrm{~nm})$ and the polydispersity index was 0.175 $( \pm 0.020)$. The Fig. 1 shows the size distribution to the PNCCA sample.

\subsection{Morphology}

The Fig. 2 presents the morphology of PNC-CA obtained by Scanning electron microscope (SEM). The image shows particles with size compatible with that obtained by Dynamic light scattering, about $200-300 \mathrm{~nm}$.
Fig. 1 Mean particle size of the PNC-CA

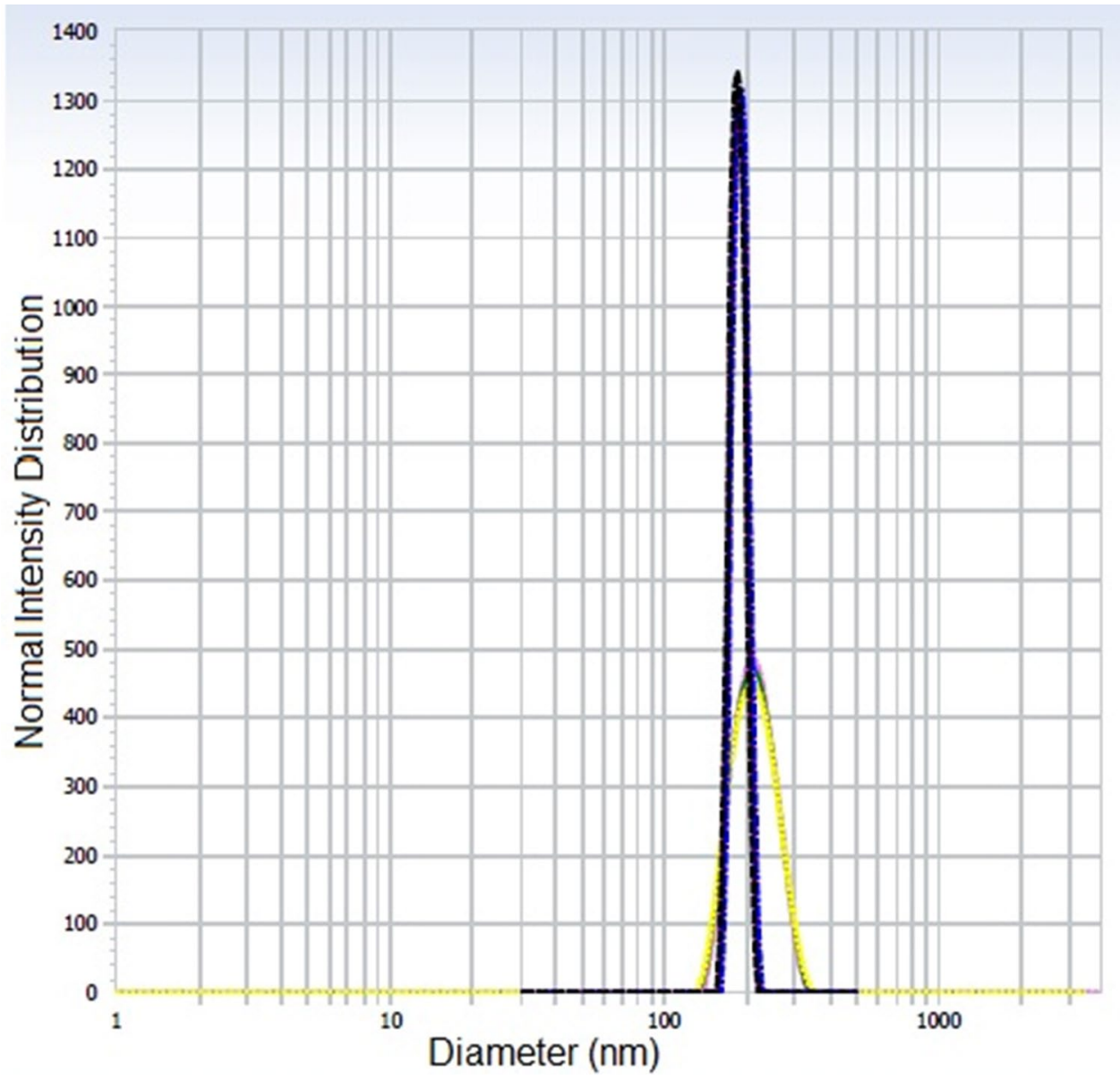

SN Applied Sciences 


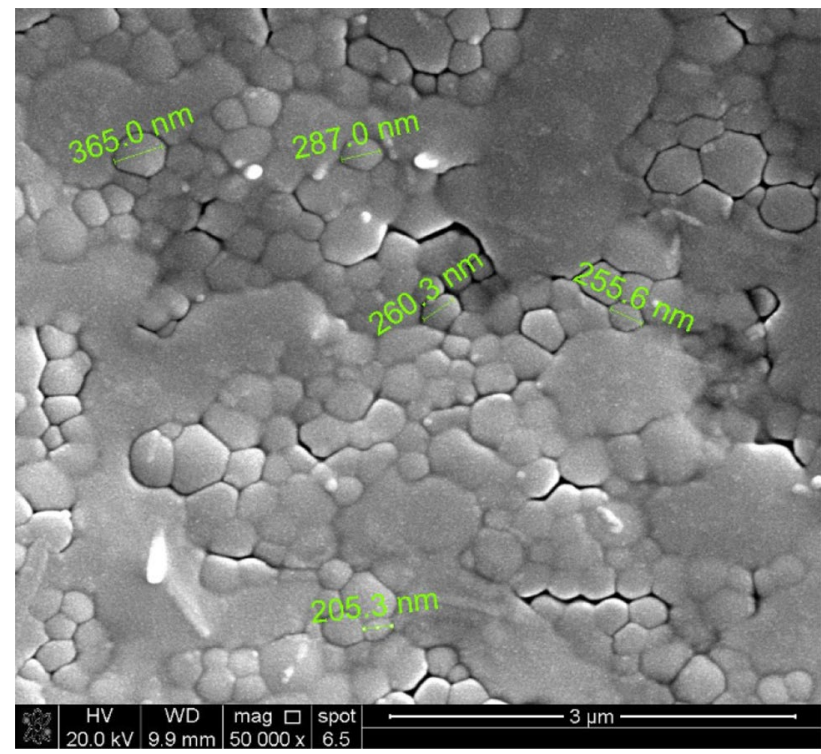

Fig. 2 SEM image of PNC-CA (magnitude of 50,000x)

\subsection{Madecassoside identification and quantification by HPLC}

The development of a HPLC method for identification and quantification of madecassoside was performed to assure the reliability of the analyses.

Through a calibration curve, it was obtained linearity in the range of $8-50 \mathrm{mg} \mathrm{L}^{-1}$, with correlation coefficient $\left(\mathrm{R}^{2}\right)$ of 0.995 . The limit of detection (LOD) and limit of quantification (LOQ) were obtained through linear regression of three calibration curves prepared according to the guidelines of the Brazil's National Health Surveillance Agency [41]. The data obtained were treated statistically with the software Excel 2010 and Action Stat version 3.7. The values of LOD e LOQ obtained were 2.62742 and $7.95369 \mathrm{mg} \mathrm{L}^{-1}$, respectively. The Fig. 3a, b show HPLC overlapped chromatograms of the standard madecassoside (in blue color), madecassoside in C. asiatica extract at 12\% (in black color) and madecassoside in PNC-CA (in red color). It was eluted
Fig. 3 HPLC chromatograms. (a) Overlapping of all chromatograms; (b) Expansion of the chromatograms. Peak 1 and 3 are interfering peaks from matrix of the CA extract; Peak 2 is the madecassoside

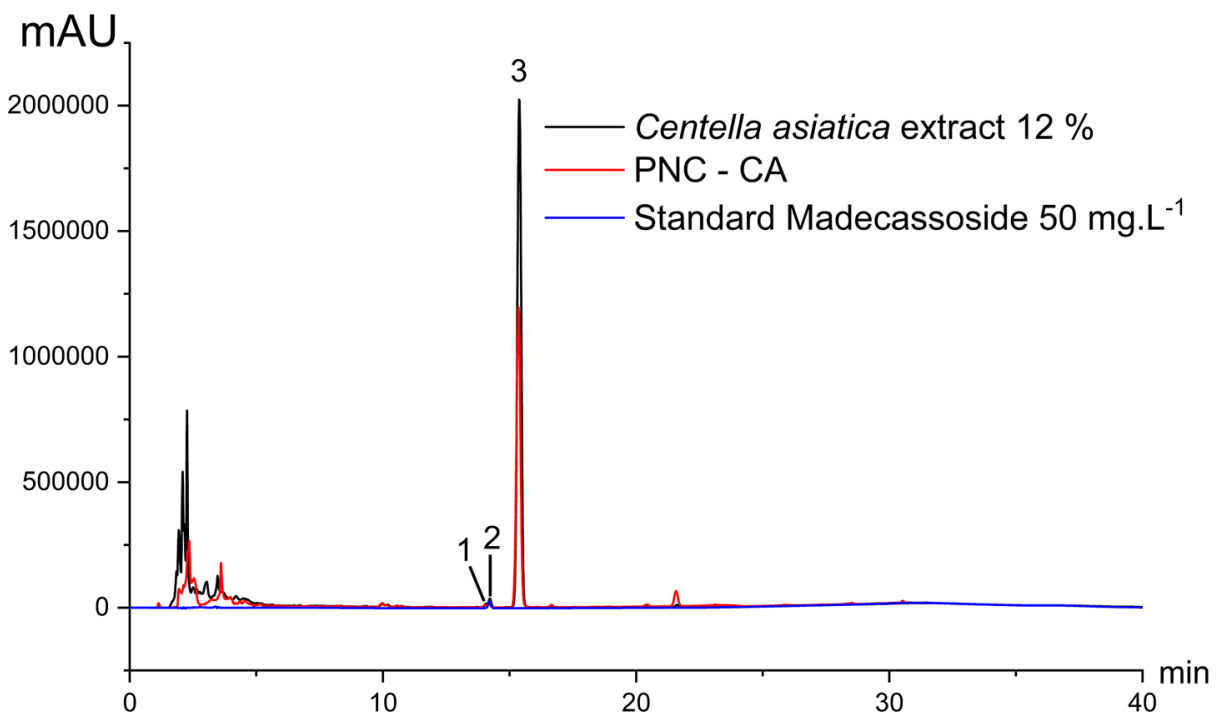

(a)

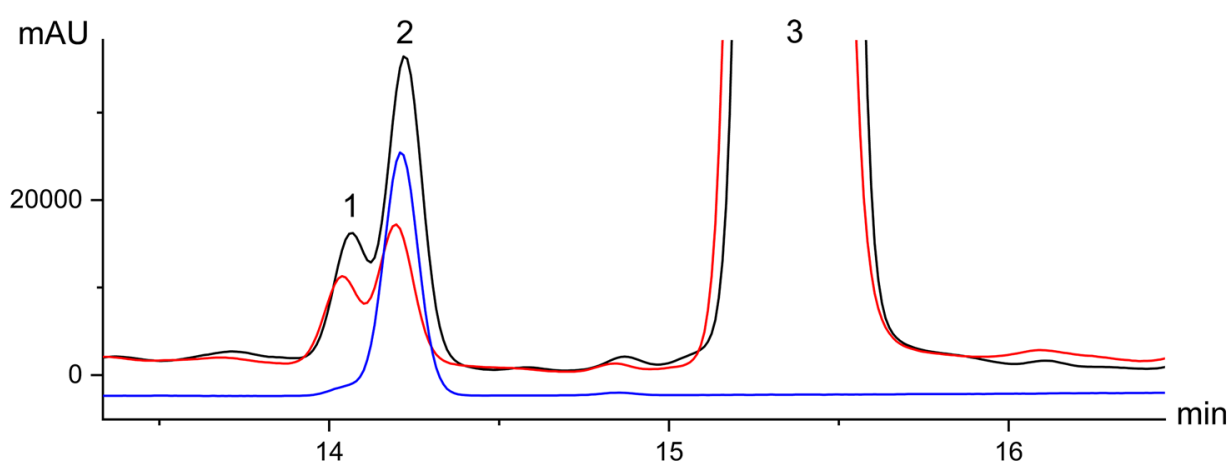

(b) 
at retention time of approximately $14.2 \mathrm{~min}$. Madecassoside concentration in C. asiatica extract and in PNCCA were $573.8 \mathrm{mg} \mathrm{L}^{-1}\left( \pm 23.4 \mathrm{mg} \mathrm{L}^{-1}\right)$ and $58.86 \mathrm{mg} \mathrm{L}^{1}$ $\left( \pm 2.21 \mathrm{mg} \mathrm{L}^{-1}\right)$, respectively. The presence of asiaticoside has been investigated but not detected in this extract.

The triterpenoids and saponins present in C.A extract are complex molecules and due to the similarity of the polarity of their structures, their separation is a challenging task [42]. The HPLC technique is widely used for the analysis of these compounds and as a device, in the chromatographic analysis the mobile phase initially has greater polarity, being composed of $80 \%$ water and $20 \%$ acetonitrile. The addition of ionic and acidic pairs can assist the separation of the RP-C18 chromatographic column. Monton et al. (2018) [43] developed an efficient method of separating madecassoside and asiaticoside using a C18-RP column with a mobile gradient phase of water and acetonitrile adding orthophosphoric acid. In previous studies [36] the chromatographic separation was performed with a column RP-C18, with mobile phase composed of water and acetonitrile with the additives trifluoroacetic acid and methyl tert-butyl ether. In this study, the researchers identified six constituents of the C.A. extract, one of them, asiacoside $B$, was described as a structural isomer of madecassoside. As a result in the chromatogram, the madecassoside peak was detected shortly after the asiaticoside B. Rafamantanana et al. (2009) [35] used water and acetonitrile gradient method using an RP-C18 column, with a peak in the chromatogram of the $C$.A. extract showing which precedes Madecassoside and partially coelutes, being called asiacoside B. Therefore, the methodology developed in this article also presents a partial coelution of an interfering peak that precedes the peak of interest of the madecassoside marker. In order to check the retention time of the madecassoside marker were done dilutions of C.A. extract which were prepared with the addition of madecassoside standard with known concentration, confirming the retention time of the marker in the chromatographic analysis.

\subsection{Entrapment efficiency}

The entrapment efficiency of the nanocarriers (EE) was $97.7 \%$, and was determined by the ratio between the concentration of encapsulated madecassoside into the nanocarriers, $\left[\right.$ Madeca $\left._{\text {encapsulated }}\right], 56.64 \mathrm{mg} \mathrm{kg}^{-1}$ and the total concentration of madecassoside in the formulation Madeca $_{\text {Total }}, 58.00 \mathrm{mg} \mathrm{kg}^{-1}$, according to Eq. (2).

\subsection{Stability of the CA entrapped in the PNC}

\subsubsection{Madecassoside content}

The stability studies were carried out at 25 and $40{ }^{\circ} \mathrm{C}$, in triplicate for each collected point. The Fig. 4 shows the

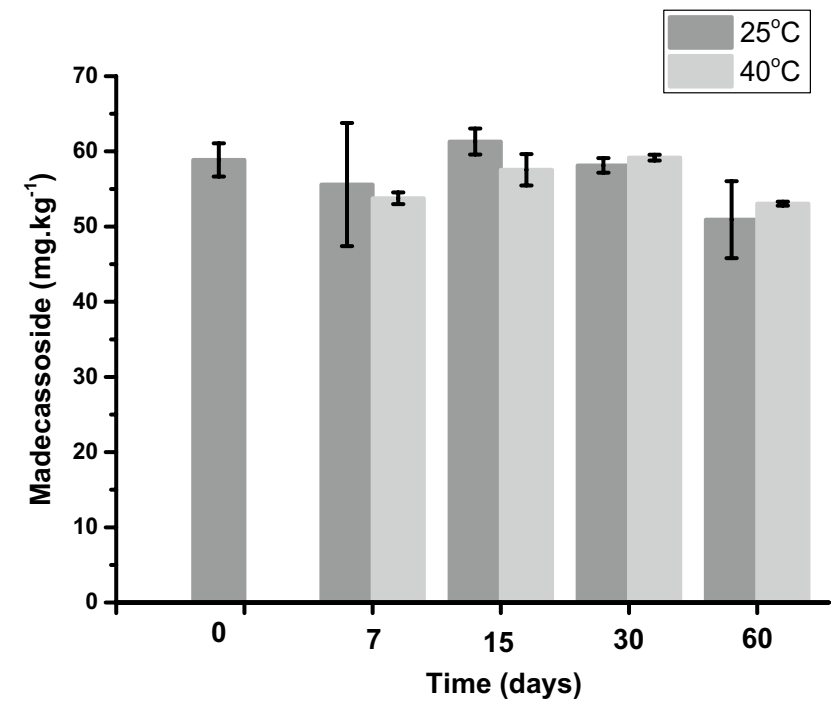

Fig. 4 Madecassoside content $\left(\mathrm{mg} \mathrm{kg}^{-1}\right)$ in the PNC-CA, under the storage conditions evaluated $\left(25\right.$ and $40^{\circ} \mathrm{C}$ ) for 60 days

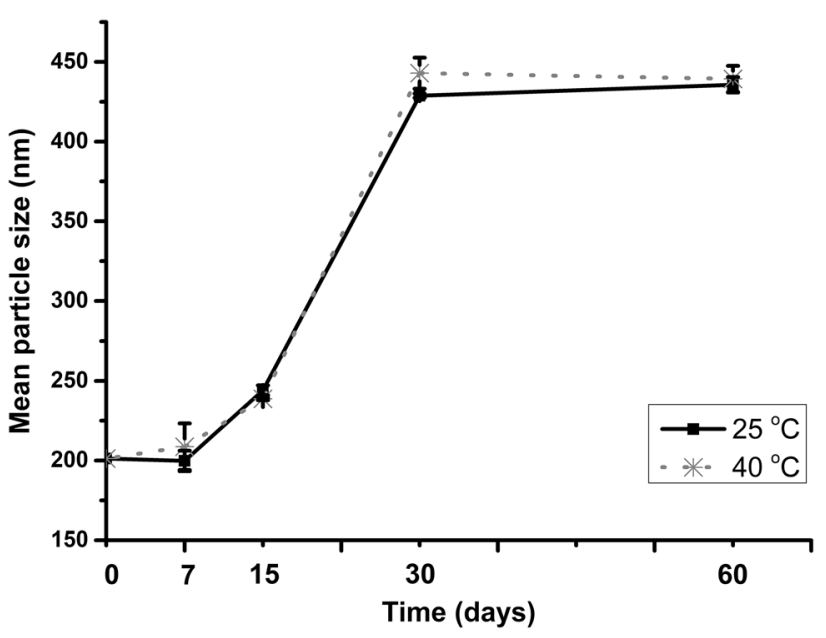

Fig. 5 Average diameter of the nanocarriers during the stability study

variation of the madecassoside concentration during the 60 days evaluated for both condition studied ( 25 and $40^{\circ} \mathrm{C}$ ).

The results show that the concentration of madecassoside remains almost constant over time, showing that the nanocarrier was effective in protecting the active in the period of 60 days, even in the extreme conditions of temperature, $40^{\circ} \mathrm{C}$.

\subsubsection{Particle size distribution}

Figure 5 shows the average diameter of the PNC-CA as a function of time. It is noted that after 15 days there is an 
increase in the average size and after that remains constant between 30 and 60 days, for both stored conditions (25 and $40^{\circ} \mathrm{C}$ ). The increase of particle average size after 15 days of stability evaluation can be associated with the increase of polydispersity index observed in both condition of studies, which varied from 0.111 to 0.264 to the store condition of $25^{\circ} \mathrm{C}$ and 0.192 to 0.294 to the store condition of $40^{\circ} \mathrm{C}$, according to the Table 1 . The increase of polydispersity index suggests an increase of non-uniformity of the size distribution of particles of colloidal system, which can affect the system stability.

\subsection{In vitro safety testing}

\subsubsection{Indirect test: Agar diffusion}

The indirect test-Agar diffusion is based on the presence of a lighter halo around the deposited test material which indicates cell death and consequently the dispersion of the dye that is retained in the cells. On the contrary, in the absence of halo around the deposited test material and dye dispersion indicate that the cells are live.

The Fig. 6a shows a macroscopic analysis of negative and positive test controls with the absence of growth inhibition halo around the atoxic paper filter and presence of lighter halo around latex glove respectively and the Fig. $6 \mathrm{~b}$ shows absence of growth inhibition halo for both samples: C. asiatica extract and PNC-CA.

Figure 7 shows the optical microscopy in the area around the tests materials. It is observed that for both C. asiatica extract and PNC-CA, the cells are morphologically normal indicating non-cytotoxicity of materials as negative control.
Table 1 Polydispersity index of PNC-CA

Fig. 6 Photograph of culture plates from indirect contact cytotoxicity assay. Negative control of cytotoxic effect: nontoxic filter paper (a, left well); positive control: piece of latex glove (a, right well); Samples of Casiatica extract (b, first line) and PNC-CA (b, second line)

\begin{tabular}{|c|c|c|c|c|c|}
\hline \multirow{2}{*}{$\begin{array}{l}\text { Storage } \\
\text { condition }\end{array}$} & \multicolumn{5}{|l|}{ Time (days) } \\
\hline & 0 & 07 & 15 & 30 & 60 \\
\hline $25^{\circ} \mathrm{C}$ & $0.163 \pm 0.048$ & $0.129 \pm 0.104$ & $0.111 \pm 0.073$ & $0.264 \pm 0.009$ & $0.211 \pm 0.058$ \\
\hline $40^{\circ} \mathrm{C}$ & $0.163 \pm 0.048$ & $0.202 \pm 0.115$ & $0.192 \pm 0.034$ & $0.294 \pm 0.029$ & $0.227 \pm 0.026$ \\
\hline
\end{tabular}
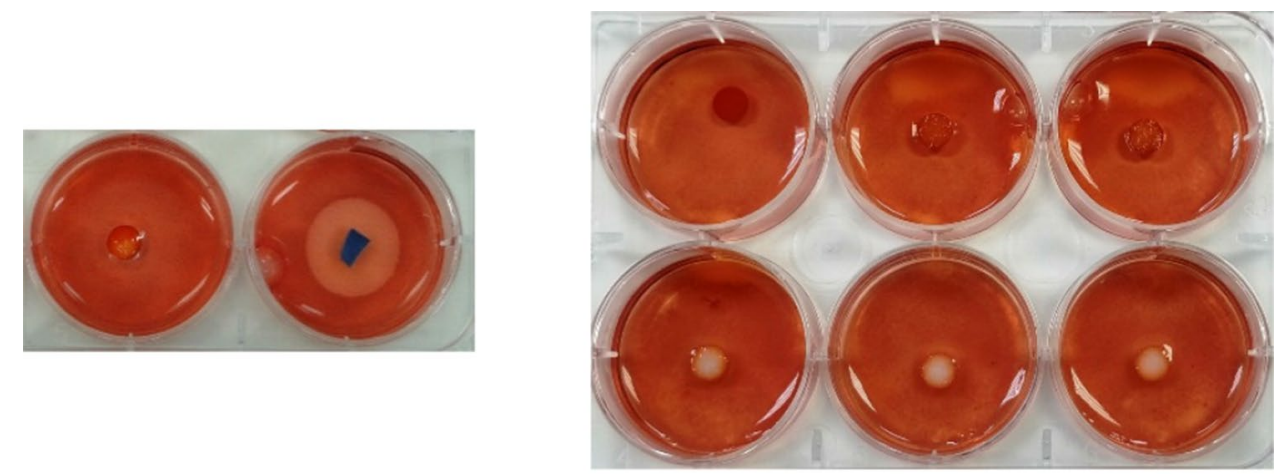

(a)

(b)

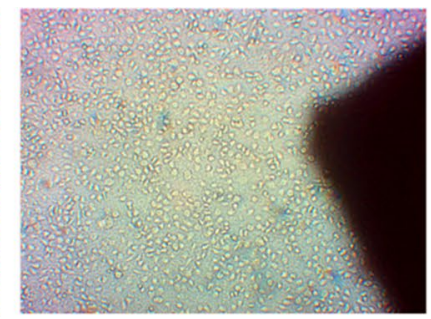

(b)

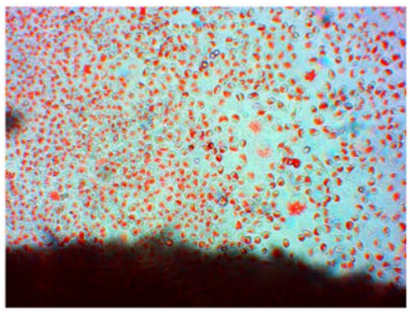

(c)

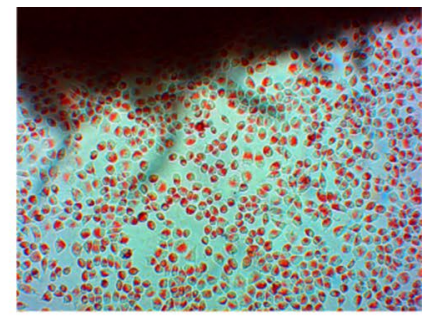

(d)
Fig. 7 Optical microscopy of cells in the area around the test materials. (a) Negative control: non-toxic filter paper; (b) Positive control of cytotoxic effect: piece of latex glove; (c) PNC-CA samples and (d) C. asiatica extract. Images from microscope Axiovert 135, Zeiss, magnification (400x)

\section{SN Applied Sciences}




\subsubsection{Cell viability}

The cell viability was evaluated after $24 \mathrm{~h}$ of contact of cells with the test materials. For the empty nanocarriers and PNC-CA the concentration was of $36 \%, 18 \%, 9 \%, 4.5 \%$, $2.3 \%, 1.1 \%, 0.6 \%$ and $0.3 \%$ and for the $C$. asiatica extract the concentration was of $4.32 \%, 2.16 \%, 1.08 \%, 0.54 \%$, $0.27 \%, 0.135 \%, 0.0675 \%, 0.03375 \%$. The results (Fig. 8 ) showed that none of the test materials were cytotoxic in the studied concentrations.

\subsubsection{Potential of cell transformation}

Aiming to complete the in vitro safety assessment tests, the cell transformation assay-CTA was performed. Figure 9 illustrates cellular aspects of the evaluated conditions. Results showed that both samples $C$. asiatica extract and encapsulated (PNC-CA) did not form type III foci, with characteristics presented by the positive control of cell transformation. Although samples formed foci when compared with the negative control condition, where there are
Fig. 8 Cell viability (\%) after $24 \mathrm{~h}$ of cell contact with the test materials

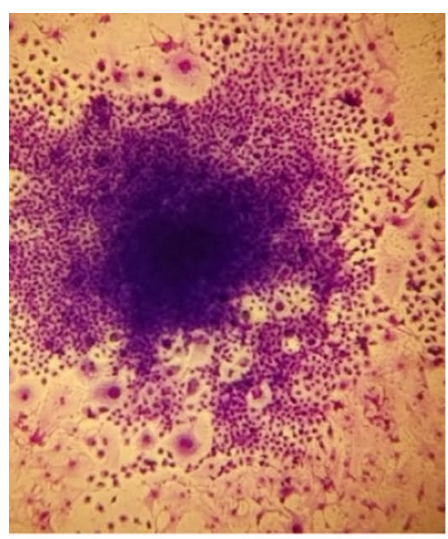

(a)

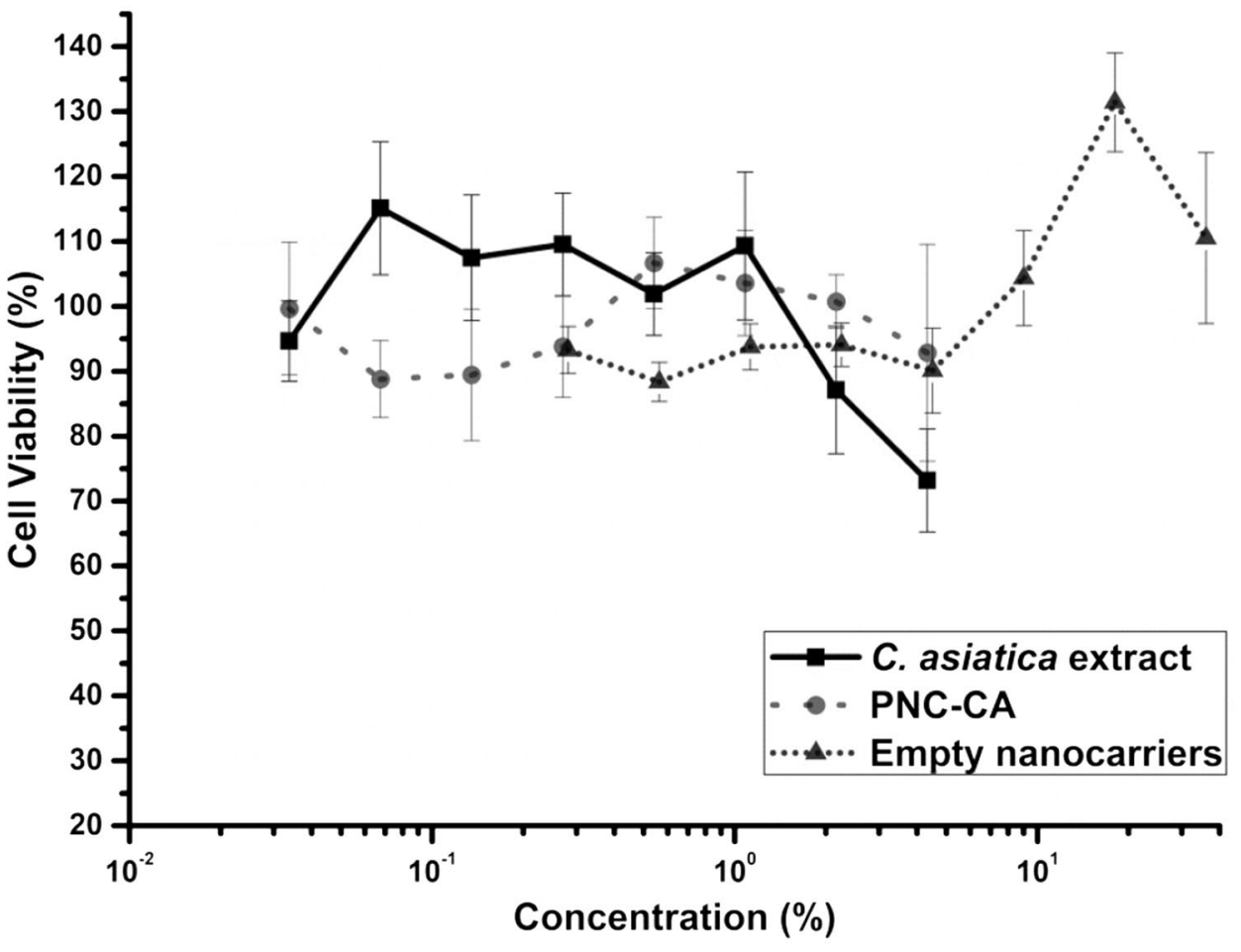

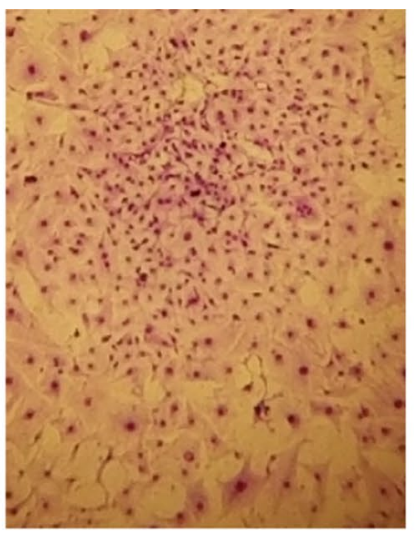

(b)

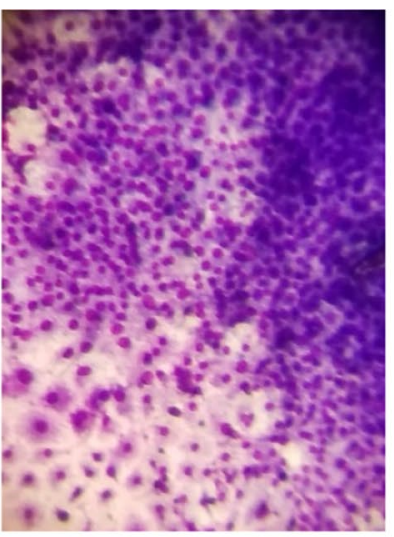

(c)

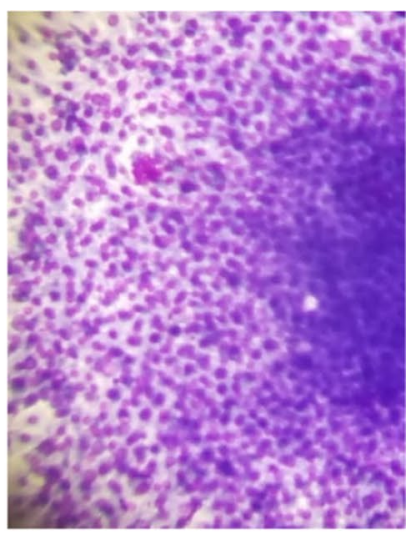

(d)
Fig. 9 Cell Transformation Assay (CTA). (a) Positive control of cell transformation: focus type III-magnification ( $8 \times)$; (b) Negative control of cell transformation magnification (8x); (c) Absence of focus type III for the cells treated with PNC-CA magnification (20x) and (d) C. asiatica extract magnification (20x). The Images were obtained using a Leica MZ8 Stereomicroscope 
cells only, and despite the basophilia, there is no piling of the cells, leaving only with focus I aspect, based on the classification proposed by Sasaki et al. [40].

Therefore, the results show that the formulations evaluated do not induce cell transformation in the in vitro assay.

\section{Discussion}

In this study, PNC-CA were prepared by emulsification process followed by high pressure homogenization, resulting in particles around $210 \mathrm{~nm}$, with PDI of 0.2 , indicating a homogeneous particle size distribution. The absence of large particles (low PDI) and the viscosity of silicone (100 CPs) can contribute to the formulation stability, once large particles are prone to aggregation.

The hydrophobic character of the formulation prepared here aims to improve its partition coefficient. Bovine serum albumin (BSA) nanoparticles [44] and transfersomes [45], both loaded with C. asiatica extract, and liposomes [27] loading only madecassoside, were prepared aiming to improve partition coefficient and, consequently, bioavailability. However, in these processes, it was used organic solvents, or chemical crosslinking agent or a non-scalable method. The nanocarriers presented in this work have the advantage of being prepared without organic solvents or chemical crooslinking agents, through a robust and scalable process.

The $C$. asiatica extract used in this work was obtained commercially and the biomarker madecassoside was identified and quantified $\left(0.57 \mathrm{mg} \mathrm{g}^{-1} \pm 0.02 \mathrm{mg} \mathrm{g}^{-1}\right)$. The presence of asiaticoside was investigated but this metabolite was not found. As reported in the literature, C. asiática is reach pentacyclic triterpenoid metabolites, like the saponins madecassoside and asiaticoside and their sapogenins (madecassic and asiatic acids), and its composition can vary depending on the plant origin, growth conditions and extraction process [46, 47]. Madecassoside was the highest triterpenoid in the extract used by Hashim (2011) [10], with $3.10 \mathrm{mg} \mathrm{mL}^{-1} \pm 4.58 \mathrm{mg} \mathrm{mL}^{-1}$, while Azaid (2016) [48] used an extract with higher asiaticoside content $\left(7.50 \mathrm{mg} \mathrm{mL}^{-1}\right)$ than madecassoside $\left(0.74 \mathrm{mg} \mathrm{mL}^{-1}\right)$, that is close to the concentration found by us.

The HPLC chromatograms (Fig. 3) showed that madecassoside in C. asiatica extract and PNC-CA had the same retention time to the standard madecassoside, confirming the presence of the extract in the nanocarriers.

These triterpenoids, madecassoside and asiaticoside, stimulate Type I collagen secretion by fibroblasts, [49], being able to minimize skin aging process, since the main mechanism is the decrease in collagen levels [50]. Furthermore, madecassoside proved to be an effective inhibitor of hyperpigmentation caused by UVB irradiation, by suppressing the production prostaglandins (PG) PGE2 and PGF2a, the main PGs produced by keratinocytes in response to UV irradiation [51].

Entrapment efficiency of $C$. asiatica extract obtained with the preparation process used here was $97.7 \%$, that is higher than the result obtained by Surini et al. [45], that prepared transferssomes containing $C$. asiatica extract by thin layer hydratation method and obtained particles around $200 \mathrm{~nm}$ and reached $85 \%$ of entrapment efficiency. Also the particle obtained in this work was higher than the nanoparticles formed as liposome type spheres by gelatin $(115 \mathrm{~nm})$, while the EE was less than $70 \%$ [26], and yet higher than the NLCs previously mentioned that reached a maximum of $45 \%$ of $E E$ for the glycolic extract of $C$. asiatica with particles size around $110 \mathrm{~nm}$ [28].

The protection of pentacyclic triterpenes against degradation provided by the polymeric nanocarriers developed in this work was effective. Madecassoside concentration remained constant over the time, in all stored conditions studied. This protection is crucial once these metabolites can degrade over time and storage conditions. Madecassoside and asiaticoside degraded approximately $20 \%$ after 16 weeks under light protected condition [52] and asiatic acid degraded after 6 months under $40^{\circ} \mathrm{C}$ [53]. Monton et al. [43] studying the stability of asiaticoside and madecassoside in filming forming polymeric dispersions, using Eudragit ${ }^{\circ} \mathrm{RS} 30 \mathrm{D}$ and Eudragit $\mathrm{RL} 30 \mathrm{D}$ as film former, concluded that madecassoside and asiaticoside content kept stable only at $4{ }^{\circ} \mathrm{C}$ storage condition, while at 25 and $40^{\circ} \mathrm{C}$, the degradation of triterpenes occur after 90 days of storage. Besides that, entrapment efficiency of $C$. asiatica glycolic extract in NLCs measured by asiaticoside concentration decreased after 30 days of storage at $4{ }^{\circ} \mathrm{C}$ from $44.7 \%$ to $34 \%$ [28].

The mean diameter of the nanocarriers during the stability studies increased between days 15 th and 30th for both temperatures, $25^{\circ} \mathrm{C}$ and, and then remained stable during the evaluation. These diameters increase observed may have occurred possible due to the aggregation phenomenon of nanoparticles which can be justified by the slightly increase of polydispersity index.

The safety assays shown that $C$. asiatica extract used here has no cytotoxic effects or carcinogenic potential on the studied concentrations. These findings are consistent with literature. Aizad et al. [48] that observed that C. asiatica extract was able to stimulate proliferation of human fibroblasts and, on the other hand, inhibited lung cancer cells (A549) growth. Pittella (2009) [54] confirmed the cytotoxic potential of $C$. asiática against mouse melanoma (B16F1), human breast cancer (MDA MB-231) and rat glioma (C6) cell lines, while no cytotoxic effects were observed in normal hamster kidney (BHK-21) cell lines. The selectivity of action could be related to the differences in 
morphology and physiology between tested cell lines, although this is not yet proven [54]. PNC-CA and empty nanocarriers also showed no cytotoxic effects on the studied concentrations.

\section{Conclusion}

In this work, polymeric colloidal nanocarriers entrapped with Centela asiatica extract were prepared for cosmetic application. The particles were prepared by a "clean" process, without the use of organic solvents or chemical cross-linking agents. The extract of Centella asiatica used had a high concentration of madecassoside, evaluated by HPLC, with methodology implemented by our group. The high encapsulation efficiency obtained (97.7\%) proves the efficiency of the process. The nanocarriers obtained here remained stable under the storage conditions studied and were able to protect madecassoside against degradation. Safety studies have shown that PNC-CAs are safe, noncytotoxic and do not induce cell transformation.

Acknowledgments The authors are grateful to the SibratecNano program, by the Fundação de Desenvolvimento da Pesquisa (FUNDEP), for the financial support.

\section{Compliance with ethical standards}

Conflict of interest The authors certify that they have no conflict of interest in the subject matter of this manuscript.

\section{References}

1. Liu MR (2008) A taxonomic evaluation of fruit structure in the family Apiaceae. University of Johannesburg, Auckland Park

2. Puttarak P, Panichayupakaranant $P$ (2012) Factors affecting the content of pentacyclic triterpenes in Centella asiatica raw materials. Pharm Biol 50:1508-1512. https://doi.org/10.3109/13880 209.2012.685946

3. Ho PJ, Sung JJ, Cheon KK, Tae HJ (2018) Anti-inflammatory effect of Centella asiatica phytosome in a mouse model of phthalic anhydride-induced atopic dermatitis. Phytomedicine 43:110 119. https://doi.org/10.1016/j.phymed.2018.04.013

4. Majeed M, Vaidyanathana P, Mundkur L et al (2016) Efficacy of Centella asiatica extract in the management of cracked feet: in vitro and clinical evidence. World J Pharm Sci 5:983-994. https ://doi.org/10.20959/wjpps201612-8213

5. Nema NK, Maity N, Sarkar BK, Mukherjee PK (2013) Matrix metalloproteinase, hyaluronidase and elastase inhibitory potential of standardized extract of Centella asiatica. Pharm Biol 51:11821187. https://doi.org/10.3109/13880209.2013.782505

6. Dwijayanti A, Frethernety A, Hardiany NS, Purwaningsih EH (2015) Hepatoprotective effects of Acalypha Indica and Centella asiatica in Rat's liver against hypoxia. Procedia Chem 14:11-14. https://doi.org/10.1016/j.proche.2015.03.003

7. Gray NE, Morré J, Kelley J et al (2014) Caffeoylquinic acids in Centella asiatica protect against amyloid- $\beta$ toxicity. J Alzheimers Dis 40:359-373. https://doi.org/10.3233/JAD-131913
8. Teerapattarakan N, Benya-aphikul H, Tansawat R et al (2018) Neuroprotective effect of a standardized extract of Centella asiatica $\mathrm{ECa} 233$ in rotenone-induced parkinsonism rats. Phytomedicine 44:65-73. https://doi.org/10.1016/j.phyme d.2018.04.028

9. Sugunabai J, Jeyaraj M, Karpagam T (2015) Analysis of functional compounds and antioxidant activity of Centella asiatica. World J Pharm Pharm Sci 4:1982-1993

10. Hashim P, Sidek H, Helan MHM, et al (2011) Triterpene composition and bioactivities of Centella asiatica. Molecules 16:13101322. https://doi.org/10.3390/molecules 16021310

11. Dewi RT, Maryani F (2015) Antioxidant and a-Glucosidase inhibitory compounds of Centella Asiatica. Procedia Chem 17:147152. https://doi.org/10.1016/j.proche.2015.12.130

12. Prakash V, Jaiswal N, Srivastava M (2017) A review on medicinal properties of Centella asiatica. Asian J Pharm Clin Res 10(10):69

13. Maramaldi G, Togni S, Franceschi F, Lati E (2014) Anti-inflammaging and antiglycation activity of a novel botanical ingredient from African biodiversity (Centevita ${ }^{\mathrm{TM}}$ ). Clin Cosmet Investig Dermatol 7:1-9. https://doi.org/10.2147/CCID.S49924

14. Yulianti L, Bramono K, Mardliyati E, Freisleben H-J (2016) Effects of Centella asiatica ethanolic extract encapsulated in Chitosan nanoparticles on proliferation activity of skin fibroblasts and keratinocytes, Type I and III collagen synthesis and aquaporin 3 expression in vitro. J Pharm Biomed Sci 6:315-327. https://doi. org/10.20936/jpbms/160246

15. Bylka W, Znajdek-Awiżeń P, Studzińska-Sroka E, Brzezińska M (2013) Centella asiatica in cosmetology. Postep dermatologii i Alergol 30:46-49. https://doi.org/10.5114/pdia.2013.33378

16. Milani M, Sparavigna A (2017) The 24-hour skin hydration and barrier function effects of a hyaluronic $1 \%$, glycerin $5 \%$, and Centella asiatica stem cells extract moisturizing fluid: an intrasubject, randomized, assessor-blinded study. Clin Cosmet Investig Dermatol 10:311-315. https://doi.org/10.2147/CCID.S144180

17. Scharffetter-Kochanek K, Brenneisen P, Wenk J et al (2000) Photoaging of the skin from phenotype to mechanisms. Exp Gerontol 35:307-316. https://doi.org/10.1016/S0531-5565(00)00098-X

18. Varani J, Dame MK, Rittie L et al (2006) Decreased collagen production in chronologically aged skin. Am J Pathol 168:1861-1868

19. Jenkins $G$ (2002) Molecular mechanisms of skin ageing. Mech Ageing Dev 123:801-810. https://doi.org/10.1016/S0047 $-6374(01) 00425-0$

20. Elsner P, Maibach H (2005) Cosmeceuticals and active cosmetics, 2nd edn. CRC Press, Boca Raton

21. Ganceviciene R, Liakou Al, Theodoridis A et al (2012) Skin antiaging strategies. Dermatoendocrinol 4:308-319. https://doi. org/10.4161/derm.22804

22. McCullough JL, Kelly KM (2006) Prevention and treatment of skin aging. Ann N Y Acad Sci 1067:323-331. https://doi.org/10.1196/ annals.1354.044

23. Saansoomchai P, Limmongkon A, Surangkul D et al (2018) Enhanced VEGF expression in hair Follicle Dermal Papilla cells by Centella asiatica Linn. C J Nat Sci 17

24. Ratz-Łyko A, Arct J, Pytkowska K (2016) Moisturizing and antiinflammatory properties of cosmetic formulations containing Centella asiatica extract. Indian J Pharm Sci 78:27-33

25. Kaul S, Gulati N, Verma D et al (2018) Role of nanotechnology in cosmeceuticals: a review of recent advances. J Pharm 2018:3420204. https://doi.org/10.1155/2018/3420204

26. Kwon MC, Choi WY, Seo YC et al (2012) Enhancement of the skin-protective activities of Centella asiatica L. Urban by a Nanoencapsulation process. J Biotechnol 157:100-106. https://doi. org/10.1016/j.jbiotec.2011.08.025

27. Wang H, Liu M, Du S (2014) Optimization of madecassoside liposomes using response surface methodology and 
evaluation of its stability. Int J Pharm 473:280-285. https://doi. org/10.1016/j.ijpharm.2014.07.010

28. da Rocha PBR, dos Santos SB, Andrade LM et al (2019) Enhanced asiaticoside skin permeation by Centella asiatica-loaded lipid nanoparticles: effects of extract type and study of stratum corneum lipid dynamics. J Drug Deliv Sci Technol 50:305-312. https ://doi.org/10.1016/j.jddst.2019.01.016

29. Yongsirasawad K, Yasurin $P$, Asavasanti $S$ et al (2018) The drug delivery system of Centella Asiatica extract-loaded gelatin nanoparticles using of one-step desolvation method. In: Proceedings of the 2018 8th International Conference on Bioscience, Biochemistry and Bioinformatics. Association for Computing Machinery, New York, NY, USA, pp 91-98

30. Huang M-S, Chanapongpisa P, Yasurin P et al (2020) Centella asiatica extract loaded BSA nanoparticles using the organic and conventional C. asiatica to improve bioavailability activity and drug delivery system. Appl Sci Eng Prog 13

31. Suwantong O, Ruktanonchai U, Supaphol P (2008) Electrospun cellulose acetate fiber mats containing asiaticoside or Centella asiatica crude extract and the release characteristics of asiaticoside. Polymer (Guildf) 49:4239-4247. https://doi.org/10.1016/j. polymer.2008.07.020

32. Sikareepaisan P, Suksamrarn A, Supaphol P (2008) Electrospun gelatin fiber mats containing a herbal-Centella asiatica-extract and release characteristic of asiaticoside. Nanotechnology 19:15102. https://doi.org/10.1088/0957-4484/19/01/015102

33. Monton C, Luprasong C, Suksaeree J, Songsak T (2020) Preparation and evaluation of film forming polymeric dispersion containing Centella asiatica extract for skin application. Adv Tradit Med. https://doi.org/10.1007/s13596-020-00451-9

34. Cerize NNP, Marim de Oliveira A, Ré MI, Tedesco AC (2011) Colloidal nanoscale carriers for active hydrophilic substances and method for producing same. WO 2011/156880 Al, 22, Dec., 2011

35. Rafamantanana MH, Rozet E, Raoelison GE et al (2009) An improved HPLC-UV method for the simultaneous quantification of triterpenic glycosides and aglycones in leaves of Centella asiatica (L.) Urb (APIACEAE). J Chromatogr B 877:2396-2402. https ://doi.org/10.1016/j.jchromb.2009.03.018

36. Schaneberg BT, Mikell JR, Bedir E, Khan IA (2003) An improved HPLC method for quantitative determination of six triterpenes in Centella asiatica extracts and commercial products. Pharmazie 58:381-384

37. Rogero SO, LugÃlpoundso AB, Ikeda TI, Cruz ÃS (2003) Teste in vitro de citotoxicidade: estudo comparativo entre duas metodologias. Mater Res 6:317-320

38. ISO DOCUMENT 10993-5 (2009) Biological evaluation of medical devices, Part 5, Tests for Cytotoxicity: In Vitro Methods

39. Vanparys P, Schechtman L, Aardema M et al (2010) Balb/c 3T3 cell transformation assay prevalidation study Report. 1-109

40. Sasaki K, Bohnenberger S, Hayashi K et al (2012) Recommended protocol for the BALB/c 3T3 cell transformation assay. Mutat Res Toxicol Environ Mutagen 744:30-35. https://doi.org/10.1016/j. mrgentox.2011.12.014

41. ANVISA (2017) RDC 166, 07/24/2017 Brasil's National Health Surveillance
42. HuaBin X, BaoGen S, YangYang W et al (2009) Separation and determination of asiaticoside, asiaticoside- $B$ and madecassoside in Centella asiatica total triterpenoid saponins by HPLC. J Liq Chromatogr Relat Technol 32:1891-1900. https://doi. org/10.1080/10826070903091597

43. Monton C, Luprasong C, Suksaeree J, SongsakT (2018) Validated high performance liquid chromatography for simultaneous determination of stability of madecassoside and asiaticoside in film forming polymeric dispersions. Rev Bras Farmacogn 28:289-293. https://doi.org/10.1016/j.bjp.2018.04.003

44. Kesornbuakao K, Yasurin P (2016) The development of Centella asiatica extract-loaded BSA nanoparticles production to improve bioavailability. Orient J Chem 32:2425-2434

45. Surini S, Djajadisastra J (2018) Formulation and in vitro penetration study of transfersomes gel containing gotu kola leaves extract (Centella Asiatica L. Urban). J Young Pharm 10:27-31

46. Mathur S, Verma RK, Gupta MM et al (2000) Screening of genetic resources of the medicinal-vegetable plant Centella asiatica for herb and asiaticoside yields under shaded and full sunlight conditions. J Hortic Sci Biotechnol 75:551-554. https://doi. org/10.1080/14620316.2000.11511284

47. James JT, Dubery IA (2009) Pentacyclic triterpenoids from the medicinal herb, Centella asiatica (L.) Urban. Molecules 14:39223941. https://doi.org/10.3390/molecules 14103922

48. Aizad S, Mohd Khairiri N, Yahaya BH, Zubairi SI (2017) A novel anti-proliferative activity (EC50) of pegaga (Centella asiatica) extract through in vitro 3-D culture microenvironment. J Teknol 79:1-10

49. Bonté F, Dumas M, Chaudagne C, Meybeck A (1995) Comparative activity of asiaticoside and madecassoside on type I and III collagen synthesis by cultured human fibroblasts. Ann Pharm Fr 53:38-42

50. Maquart FX, Chastang F, Simeon A et al (1999) Triterpenes from Centella asiatica stimulate extracellular matrix accumulation in rat experimental wounds. Eur J Dermatol 9:289-296

51. Jung E, Lee J-A, Shin S et al (2013) Madecassoside inhibits melanin synthesis by blocking ultraviolet-induced inflammation. Molecules 18:15724-15736. doi: https://doi.org/10.3390/molec ules181215724

52. Puttarak $P$, Brantner $A$, Panichayupakaranant $P$ (2016) Biological activities and stability of a standardized pentacyclic triterpene enriched Centella asiatica extract. Nat Prod Sci 22:20-24

53. Kaur I, Suthar N, Kaur J et al (2016) Accelerated stability studies on dried extracts of Centella asiatica through chemical, HPLC, HPTLC, and biological activity analyses. J Evid Based Complement Altern Med 21:NP127-NP137. https://doi. org/10.1177/2156587216661468

54. Pittella F, Dutra RC, Junior DD et al (2009) Antioxidant and cytotoxic activities of Centella asiatica (L) Urb. Int J Mol Sci 10:37133721. https://doi.org/10.3390/ijms10093713

Publisher's Note Springer Nature remains neutral with regard to jurisdictional claims in published maps and institutional affiliations. 